\title{
OPEN PROBLEMS ON UNIVALENT AND MULTIVALENT FUNCTIONS
}

\author{
BY A. W. GOODMAN
}

1. Introduction. Let $f(z)$ be regular in the unit circle $\varepsilon:|z|<1$, and represented by the power series

$$
w=f(z)=\sum_{n=0}^{\infty} b_{n} z^{n}=b_{0}+b_{1} z+b_{2} z^{2}+\cdots .
$$

The function $f$ maps $\varepsilon$ onto some subdomain $\delta$ of a Riemann surface, and $S$ is determined by the sequence $\left\{b_{n}\right\}$ of coefficients in (1.1).

Many questions (both open and settled) can be classified as special cases of the two general questions:

Given a geometric property of $s$ what can be said about the sequence $\left\{b_{n}\right\}$ ? Given some information about the sequence $\left\{b_{n}\right\}$, what can be said about the domain $S$ ?

One geometric property of $S$ is specified by saying that $f(z)$ is univalent in $\varepsilon$. By definition, $f(z)$ is univalent in $\varepsilon$ if

$$
f\left(z_{1}\right)=f\left(z_{2}\right), \quad z_{1}, z_{2} \in \varepsilon \Rightarrow z_{1}=z_{2} .
$$

Briefly, $f(z)$ is univalent in $\varepsilon$ if it assumes no value more than once for $z$ in $\varepsilon$. When $f(z)$ is univalent, the image of $\varepsilon$ forms a simple domain in the w-plane. The concept of univalence has a natural extension as described in

DEFINITION 1. Let $p$ be a natural number. The function $f(z)$ is said to be p-valent (or multivalent of order $p$ ) in $\varepsilon$ if the conditions

$$
f\left(z_{1}\right)=f\left(z_{2}\right)=\cdots=f\left(z_{p+1}\right), \quad z_{1}, z_{2}, \cdots, z_{p+1} \in \varepsilon
$$

imply that $z_{j}=z_{k}$ for some pair such that $j \neq k$, and if there is some $w_{0}$, such that the equation $f(z)=w_{0}$ has $p$ roots (counted in accordance with their multiplicities) in $\varepsilon$.

In brief, $f(z)$ is $p$-valent in $\varepsilon$ if it assumes no value more than $p$ times in $\varepsilon$, but assumes some value $p$ times in $\varepsilon$. We let $V(p)$ denote the class of all functions that are regular and $p$-valent in $\varepsilon$, and have $f(0)=0$.

Certain related classes are also of interest. We let $\Re(p)$ denote the subclass of $V(p)$ of those functions $f$ for which $f(\varepsilon)$ is (in a generalized

\footnotetext{
1 An expanded version of an address delivered to the Society in Tampa on November 11, 1966, by invitation of the Committee to Select Hour Speakers for the Southeastern Sectional meeting; received by the editors July 11, 1968.
} 
sense) convex. Let $S \Im(p)$ be the subclass of $V(p)$ for which $f(\varepsilon)$ is starlike with respect to some point, and let $S J^{(0)}(p)$ be the subclass for which $f(\mathcal{E})$ is starlike with respect to the origin. The class $R(p)$ consists of those functions in $U(p)$ for which all of the coefficients are real. The class $\Im(p)$ consists of those functions [25], [56] that are typicallyreal of order $p$ in $\varepsilon$. Finally we let $P$ denote the class of functions

$$
g(z)=1+\sum_{n=1}^{\infty} c_{n} z^{n}
$$

that are regular in $\varepsilon$, and have positive real part in $\varepsilon$.

2. The literature. One usually assigns the beginning of the theory of univalent functions to 1907 and the paper by Koebe [34]. The first respectable surveys of the literature on univalent and multivalent functions were given in 1933 by Montel [45] and in 1938 by Biernacki [6]. Subsequently, the volume of material increased in the usual exponential manner, and it was difficult for the beginner to determine the current status of any given problem. But recently this normal state of affairs has been temporarily reversed. Books by Schaeffer and Spencer [58], and Jenkins [33] cover in great detail special areas in the field. The texts by Hayman [28] and Goluzin [18] give a comprehensive survey, and Chapter 6 of Research problems in function theory [31] contains enough open problems in the theory of univalent and multivalent functions to occupy our efforts for some time. Further guidance in the field is supplied by the survey articles of Bernardi [4], Krzyz [36] and Hayman [30].

Finally, mention must be made of the exhaustive bibliography prepared by Bernardi [5]. This list contains 1,694 references covering the publications of at least 570 authors who work in the theory of univalent and multivalent functions. Compiling this list must have been a laborious and unrewarding task. Nevertheless, the excellent arrangement, and clever indexing, make it a welcome and valuable aid to the serious mathematicians concerned with this field.

Any report on open problems must duplicate some of the material contained in the surveys cited above, but we will try to hold the duplication to a minimum.

3. Coefficient problems. If $f(z) \in \mathcal{V}(1)$ then $f^{\prime}(z) \neq 0$ in $\varepsilon$. Consequently $b_{1} \neq 0$ in (1.1) and it is customary to normalize the function by replacing $f(z)$ with

$$
g(z) \equiv\left(f(z)-b_{0}\right) / b_{1}=z+\sum_{n=2}^{\infty} a_{n} z^{n} .
$$


Clearly $g(z)$ is regular and univalent in $\mathcal{E}$, if and only if $f(z)$ is. We let $V_{1}(1)$ be the subclass of $V(1)$ of functions such that $f^{\prime}(0)=1$.

It has been widely conjectured that if $g(z) \in \mathcal{V}_{1}(1)$, then

$$
\left|a_{n}\right| \leqq n, \quad n=2,3, \cdots .
$$

This conjecture has been proved for $n=2,3$, and 4 , and in a large variety of special cases $[4],[5],[18],[28],[33],[45],[58]$ but in general the inequality (3.2) still poses an open problem. If the bound on the right side were proved, it would be best possible (sharp) because the Koebe function

$$
u(z) \equiv \frac{z}{(1-z)^{2}}=z+\sum_{n=2}^{\infty} n z^{n}
$$

is in $v_{1}(1)$. It is easy to prove that $u(z)$ maps $\varepsilon$ onto the domain formed by deleting the points $z \leqq-\frac{1}{4}$ from the complex plane. The conjecture that $u(z)$ supplies the maximum for $\left|a_{n}\right|$ is a natural consequence of the remark that $u(\mathcal{E})$ is a maximal simple domain (if any open set is adjoined to $u(\varepsilon)$ then the new domain is no longer simple).

Now the question posed by (3.2) can be varied in many interesting ways. Let $G$ be some fixed geometric property that determines a family of functions $\mathfrak{F}_{1}(G)$ in the sense that $g(z) \in \mathfrak{F}_{1}(G)$ if and only if $g(z)=z+\sum_{2}^{\infty} a_{n} z^{n} \in V_{1}(1)$ and $g(\varepsilon)$ has the geometric property $G$. These conditions automatically determine a sequence of numbers $\left\{\phi_{n}(G)\right\}$ such that if $g(z) \in \mathscr{F}_{1}(G)$, then

$$
\left|a_{n}\right| \leqq \phi_{n}(G), \quad n=2,3, \cdots .
$$

Here it is understood that $\phi_{n}(G)$ is the smallest number such that (3.4) holds for every $g(z)$ in $\Im_{1}(G)$.

As examples of suitable geometric properties we have

$G_{1} . g(z)$ is bounded in $\mathcal{E}$.

$G_{2} . g(\varepsilon)$ is a convex domain.

$G_{3} . g(\varepsilon)$ is starlike with respect to $w=0$.

$G_{4} . g(\varepsilon)$ omits some specified set of points.

$G_{5} . g(\varepsilon)$ covers some fixed set of points.

$G_{6} . g(\varepsilon)$ has a boundary curve of fixed length.

The bounds in (3.4) are well known for $G_{2}$ and $G_{3}$. Indeed, Loewner [40] proved that $\phi_{n}\left(G_{2}\right)=1$ and Nevanlinna [46] proved that $\phi_{n}\left(G_{8}\right)=n$. Only partial results have been obtained for $\phi_{n}\left(G_{k}\right)$ for $k=1,4$, and 5 , and as far as I am aware the determination of $\phi_{n}\left(G_{6}\right)$ has not been touched. The reader can easily add other geometric properties to the above list. However, it is not an easy task to find a $G$ that is new, interesting, and for which some of the numbers $\phi_{n}(G)$ can be computed explicitly. 
We now turn our attention to $\vartheta(p)$. The theory of $p$-valent functions is much more than just a generalization of the theory of univalent functions. If $T$ is a theorem about the set $V(1)$, the extension to $U(p)$ for $p \geqq 2$, may be completely trivial, or extremely difficult, or perhaps false. Merely to cite one difficulty the normalization $g(z)=z+\cdots$ is not even possible for 2 -valent functions. Further the subclass $v_{1}(2)$ of 2-valent functions of this form is not compact. Indeed, given $M$, arbitrarily large, and $n \geqq 2$, a fixed positive integer, it is easy to show that there is a $g$ in $v_{1}(2)$ of the form (3.1) for which $\left|a_{n}\right|>M$. More generally [19] if we fix any $p-1$ of the first $p$ coeffcients in

$$
f(z)=b_{1} z+b_{2} z^{2}+\cdots+b_{p} z^{p}+\sum_{n=p+1}^{\infty} b_{n} z^{n},
$$

then it is still possible to find an $f$ in $V(p)$ with the specified $p-1$ coefficients and with $b_{n}$ arbitrarily large for each $n>p$. Consequently, for a natural extension of the conjecture $\left|a_{n}\right| \leqq n$, we require that the first $p$ coefficients in (3.5) be fixed (although there are other restrictions that are also of interest). After some labor $\mathrm{we}^{2}$ arrive at the following

CONJECTURE. Let $p \geqq 2$ be a fixed integer and let $f(z)$ be given by (3.5). If $f(z) \in \mathcal{V}(p)$, then for each $n>p$

$$
\left|b_{n}\right| \leqq \sum_{k=1}^{p} \frac{2 k(p+n) !}{(p+k) !(p-k) !(n-p-1) !\left(n^{2}-k^{2}\right)}\left|b_{k}\right|
$$

Briefly, the conjecture is that the $n$th coefficient is bounded by a certain linear combination of the first $p$ coefficients. It has been proved [19] that if the bound on the right side of (3.6) is true then it is the sharp bound, because for each set $\left\{B_{1}, B_{2}, \cdots, B_{p}\right\}$ of nonnegative numbers, not all zero, there is an $f$ in $U(p)$ for which $\left|b_{k}\right|=B_{k}$ for $k=1,2, \cdots, p$, and for which we have (3.6) with the equality sign for each $n>p$. The simplest case of (3.6) occurs when $p=2$ and $n=3$. Then the conjecture (3.6) becomes

$$
\left|b_{3}\right| \leqq 5\left|b_{1}\right|+4\left|b_{2}\right| \text {. }
$$

Using variational methods $\mathrm{M}$. Watson [61] has proved a number of interesting theorems on 2-valent functions that tend to strengthen the conjecture (3.7), but even this simple inequality still poses an open problem.

2 This conjecture was first proposed in the author's thesis [19]. 
If we look at certain subclasses of $v(p)$ then we can go much further with (3.6). Indeed, it was proved in [25] that if $f(z) \in S J^{(0)}(p) \cap R(p)$, then the sharp inequality (3.6) holds for each $n>p \geqq 1$ and every set $\left\{\left|b_{1}\right|,\left|b_{2}\right|, \cdots,\left|b_{p}\right|\right\}$ of nonnegative numbers. In fact the same proof gives the same sharp result for the larger class of functions $J(p)$. Later Gel'fer [14] obtained the same result with some moderate extensions.

This line of investigation was continued by M. S. Robertson [55] who proved the following remarkable result.

TheOREM 1. Let

$$
f(z)=\sum_{-\infty}^{\infty} a_{n} z^{n}
$$

be regular in $\rho \leqq|z|<1$, and let $p$ be a fixed positive integer. If on each circle $|z|=r, \rho \leqq r<1$, the imaginary part of $f(z)$ changes sign $2 p$ times, then for each $n>p$

$$
\begin{aligned}
\left|a_{n}-\bar{a}_{-n}\right| \leqq & \frac{(n+p) !}{n(p !)^{2}(n-p-1) !}\left|a_{0}-\bar{a}_{0}\right| \\
& \quad+2 \sum_{k=1}^{p} \frac{n(n+p) !\left|a_{k}-\bar{a}_{-k}\right|}{\left(n^{2}-k^{2}\right)(p+k) !(p-k) !(n-p-1) !}
\end{aligned}
$$

and these bounds are sharp in all of the variables.

From this theorem, Robertson deduces the inequality (3.6) for another class $g(p)$ of $p$-valent functions which neither contains $J(p)$ nor is contained in $J(p)$. The class $g(p)$ is the natural extension to $p>1$ of the class of univalent functions that are convex in the direction of the imaginary axis. It is worth noting that the bound (3.6) is thus established for a large class of $p$-valent functions in which the coefficients $b_{n}$ may be complex. These results for the classes $J(p)$ and $g(p)$ represent the present outer limits of our knowledge about the conjecture that (3.6) holds for all $f$ in $v(p)$.

If we assume in addition that $f(z)$ has $p$ zeros at the origin, then $f(z)$ can be put in the form

$$
f(z)=z^{p}+b_{p+1} z^{p+1}+b_{p+2} z^{p+2}+\cdots \cdot
$$

For this special subclass of $\mathcal{V}(p)$, Hayman ${ }^{3}$ [26] has proved that

3 After this paper was completed Professor Hayman informed me that D.C. Spencer obtained the same result: On mean one-valent functions, Ann. of Math. (2) 42 (1941), 614-633. This inequality was also proved by G. M. Goluzin, On p-valent functions, Mat. Sb. 8(50) (1940), 277-284. (Russian) 
$\left|b_{p+1}\right| \leqq 2 p$ and Jenkins [33] has proved that $\left|b_{p+2}\right| \leqq p(2 p+1)$. Both of these results are consistent with (3.6), but beyond these no other sharp results are known. Asymptotic results and various estimates for the coefficients are covered by Hayman in [27], [28] and the reader is referred to these sources for further details.

Variational methods have been applied to the coefficient problem for multivalent functions, first by Schiffer [59] and later by Gel'fer [15], [16], [17], but as far as I am aware, the results are only qualitative, and somewhat disappointing.

4. The influence of zeros on the coefficients. Biernacki [6, p. 28] was the first to point out in print that the number of zeros of $f(z)$ has an influence on the magnitude of the coefficients, and indeed he proved that for each $p \geqq 1$ there is a constant $C(p)$ such that if $f(z) \in \mathcal{V}(p)$ and has $s$ zeros in $\mathcal{E}$, then for $n>s$

$$
\left|b_{n}\right| \leqq C(p) \max \left\{\left|b_{1}\right|,\left|b_{2}\right|, \cdots,\left|b_{s}\right|\right\} n^{2 p-1} .
$$

No suggestion for the sharp upper bound in (4.1) was made until 1951 when the author $[21]$ proposed the following. Let

$$
f(z)=z^{q}+\sum_{n=q+1}^{\infty} b_{n} z^{n}, \quad q \geqq 1
$$

be in $V(p)$, and suppose that in addition to the $q$ th order zero of $f(z)$ at the origin, $f(z)$ has $t$ zeros $\beta_{1}, \beta_{2}, \cdots, \beta_{t}$ in $0<|z|<1$, where $q+t=s \leqq p$. Define $B_{n}$ by

$$
\begin{aligned}
F(z) & \equiv \frac{z^{q}}{(1-z)^{2 q}}\left(\frac{1+z}{1-z}\right)^{2(p-s)} \frac{1}{(1-z)^{2 t}} \prod_{j=1}^{t}\left(1+\frac{z}{\left|\beta_{j}\right|}\right)\left(1+\left|\beta_{j}\right| z\right) \\
& \equiv z^{q}+\sum_{n=q+1}^{\infty} B_{n} z^{n} .
\end{aligned}
$$

Then the conjecture is that for each $n>q$

$$
\left|b_{n}\right| \leqq B_{n} \text {. }
$$

The inequality (4.4) has been proved if $f(z) \in J(p)$, but although the conjecture was repeated in [22], there has been little advance beyond this. It should be pointed out that the two conjectures embodied in (3.6) and (4.4) both specialize to $\left|b_{n}\right| \leqq n$, when $p=1(f(z)$ is univalent). Consequently, we have natural generalizations in two distinctly different directions for the coefficient problem for univalent functions. 
Both of these conjectures suggest allied conjectures concerning bounds for $|f(z)|,\left|f^{\prime}(z)\right|$ etc., but in the interest of brevity we will omit these items.

5. Starlike functions. If in (4.3) $q+t=p$, then (4.3) can be put in the form

$$
\begin{aligned}
F(z) & =\frac{z^{q}}{(1-z)^{2 p}} \prod_{j=1}^{p-q}\left(1+\frac{z}{\left|\beta_{j}\right|}\right)\left(1+\left|\beta_{j}\right| z\right) \\
& \equiv z^{q}+\sum_{n=q+1}^{\infty} B_{n}^{(p)} z^{n} .
\end{aligned}
$$

In the special case the conjecture

$$
\left|b_{n}\right| \leqq B_{n}^{(p)}, \quad n>q,
$$

is a little more tractable. It has been proved $[20$, p. 214] that if $f(z)$ is given by (4.2), has $t=p-q$ zeros $\beta_{1}, \beta_{2}, \cdots, \beta_{t}$ in $0<|z|<1$, and is in $\delta J^{(0)}(p)$, then (5.2) holds for each $n>q$. This same result was obtained later in a somewhat simpler way by Bender [3] and has been extended and generalized in a variety of ways [5]. The class $S \Im^{(0)}(p)$ should be approached cautiously. Hummel [32] distinguishes six different ways of defining a starlike $p$-valent function. Although some of these definitions are then shown to be equivalent, Hummel proves that for $p>1$ these definitions determine three different classes of functions, each worthy of the title starlike. Even for $p=1$, these definitions lead to two different classes of starlike functions.

Is the bound (5.2) sharp? It turns out that with four of the definitions for a starlike multivalent function, (5.2) is sharp, but as pointed out by Sakaguchi and Watanabe $[57$, p. 6] $(5.2)$ gives only an upper bound for the remaining two classes of starlike multivalent functions, and the sharp upper bound in each of these cases is still unknown despite an erroneous claim to the contrary [20, p. 214].

B. N. Rahmanov [51] replaces the radial line segments by arcs of other curves, and obtains six different generalizations of the concept of a plane starlike domain to plane domains of a more complicated type. For each of these generalizations, Rahmanov finds a condition on the mapping function that corresponds to the classical one that $R\left[z f^{\prime}(z) / f(z)\right]>0$ for $f(z)$ in $S J^{(0)}(1)$.

Each convex function $h(z)$ is paired with some starlike function $f(z)$ by the Alexander relation: $f(z)=z h^{\prime}(z)$. Consequently, definitions, theorems, and conjectures for starlike functions generate corresponding definitions, theorems, and conjectures for convex functions, and hence we may omit these later items. 
6. Stieltjes integral representation. It is well known that $g(z)$ $=1+\sum_{1}^{\infty} c_{n} z^{n} \in \mathcal{P}$, if and only if there is a $\mu(t)$ that is nondecreasing in $[0,2 \pi]$ such that

$$
g(z)=\int_{0}^{2 \pi} \frac{1+e^{-i t} z}{1-e^{-i t_{z}}} d \mu(t), \quad \int_{0}^{2 \pi} d \mu(t)=1 .
$$

From (6.1) it is easy to show that $\left|c_{n}\right| \leqq 2$ and to deduce a variety of other properties of $g(z)$. The representation (6.1) for $g(z)$ is a rich source for theorems. The technique involves finding or creating a class of functions $X$ that is related in some nice way to the class $P$. Then results about $\beta$, obtained from (6.1), imply interesting results about $x$. This method has been widely exploited [5], in particular when $x=\delta J^{(0)}(1), \mathscr{K}(1)$, and $J(1)$.

From a theoretical point of view, the representation (6.1) contains implicitly all that we may wish to know about the class $P$, but from a practical point of view it is not always a trivial matter to obtain the information we wish. For example, let $\beta^{\prime}$ be the class of functions $f(z)=z+\cdots$ for which $f^{\prime}(z) \in \odot$. The Wolff-Noshiro-Warschawski Theorem implies that if $f(z) \in \mathcal{P}^{\prime}$, then $f(z)$ is univalent in $\varepsilon$. One might conjecture (as did Zmorovic [63]) that if $f(z) \in \sigma^{\prime}$, then $f(z)$ $\in S \Im^{(0)}(1)$. Gal'perin $\left[13\right.$, p. 51] asserted that there is an $f(z) \in \mathcal{P}^{\prime}$ that is not in $S J^{(0)}(1)$, and Zmorovič [64, p. 178] and later $\mathrm{Krzyz}$ [35] gave explicit examples.

These negative results leave open the problem of finding $R_{s}$, the radius of starlikeness for the class $\boldsymbol{\rho}^{\prime}$. By definition $R_{8}$, for a class of functions $\mathcal{F}$, is the largest $R$ with the property that every $f(z)$ in $\mathcal{F}$ is starlike with respect to the origin for $|z|<R$. Similarly $R_{\mathrm{e}}$, the radius of convexity for a class $\mathcal{F}$, is the largest $R$ such that every $f(z)$ in $\mathcal{F}$ is convex in $|z|<R$. Although $R_{s}$ is unknown for the class $\Phi^{\prime}$, MacGregor [42] has proved that $R_{\mathrm{o}}=2^{1 / 2}-1$ for $\boldsymbol{P}^{\prime}$.

7. Critical points. A critical point of $f(z)$ is a point at which $f^{\prime}(z)$ is zero. The presence of a single critical point is sufficient to destroy the univalence of $f(z)$.

In contrast, a function may have infinitely many critical points in $\varepsilon$ and still be 2-valent in $\varepsilon$ (see [23]). However, these critical points cannot crowd about the origin too closely but are governed by

TheOREM 2. For each positive integer $p$ and for each integer $k \geqq p$ there is a positive number $R^{\star}(p, k)$ with the following properties:

A. Let $f(z)$ be regular in $\&$ and have critical points $C_{1}, C_{2}, \cdots, C_{k}$ in 8 , where multiple critical points are listed in accordance with their multiplicity. If 


$$
\left|C_{j}\right| \leqq R^{\star}(p, k), \quad j=1,2, \cdots, k
$$

and if for at least one index $j$ the strict inequality holds, then $f(z)$ has valence greater than $p$ in $\varepsilon$.

B. The number $R^{\star}(p, k)$ is the largest number with the property $\mathrm{A}$.

The conjecture advanced in [22] is that

$$
R^{\star}(p, k)=R(p, k) \equiv\left[2 p-1-2\left(p^{2}-p\right)^{1 / 2}\right]^{1 / k}
$$

and is based partly upon the fact that the function

$$
F(z)=z\left(1-z^{k}\right)^{(2 p-2) / k} /\left(1+z^{k}\right)^{2 p / k}
$$

is $p$-valent in $\varepsilon$ and has precisely $k$ simple critical points, each with modulus $R(p, k)$. Aside from the results obtained in [22], nothing else seems to be known about $R^{\star}(p, k)$.

8. A special class of meromorphic functions. Let $\delta$ be a set of points and let $\mathcal{F}(S)$ be the family of functions of the form

$$
f(z)=\sum_{k=1}^{n} \frac{A_{k}}{z-a_{k}}
$$

where $A_{k}>0$ and $a_{k} \in \mathcal{S}, k=1,2, \cdots, n$. Čakilov [8], [9] first raised the question of finding the domain of univalence for the class $F(S)$, and answered it in two interesting cases: (a) If $S$ is the interval $-1 \leqq z \leqq 1$, then each $f$ in $\Im(S)$ is univalent in $|z|>1$, and this domain is maximal. (b) If $S$ is the disk $|z| \leqq 1$, then each $f$ in $\mathcal{F}(\mathcal{S})$ is univalent in $|z|>\sqrt{2}$, and this domain is maximal. These results of Cakilov have been extended in various ways [1], [2], [11], [60].

$R$. Distler [10] found the precise domain of univalence for the family $\mathcal{F}(\mathcal{S})$, for each arbitrary point set $\mathcal{S}$. Let $\mathfrak{C}$ be the closed convex cover of $S$ and let $D$ be the director set of $\mathcal{C}$. By this we mean the set of points $P$ such that $\mathcal{C}$ subtends an angle of $\pi / 2$ at $P$. The set $D$ may be empty, but if $\delta$ is a bounded set with at least two points, then $D$ will be a simple closed curve. Distler's theorem then states that if $f \in F(S)$ then $f$ is univalent in the domain outside of the director curve $D$, and this domain is maximal. Since the director set of the line segment $-1 \leqq z \leqq 1$ is the circle $|z|=1$, and the director set of the circle $|z|=1$ is the circle $|z|=2^{1 / 2}$, Distler's theorem contains the two Cakalov theorems as special cases. Distler's proof is very simple, and can be generalized to include the case in which the coefficients $A_{k}$ are complex, but are restricted to lie in some sector.

One naturally asks for the domain of $p$-valence for the family $\mathcal{F}(\$)$, and as far as I am aware, this problem is open for $p \geqq 2$. Also untouched are questions on the starlikeness or convexity of the image of $|z|=R$ under functions in the family $F(S)$. 
9. Sums and products. Let $\mathfrak{F}$ be some class of functions such as $\mathcal{V}(1), \delta J^{(0)}(1), \mathcal{K}(1)$, etc., regular in $\mathcal{E}$ and let $\mathcal{F}_{1}$ be the subclass of functions with the representation

$$
f(z)=z+\sum_{n=2}^{\infty} a_{n} z^{n} .
$$

We let $Q$ be the class of all functions $\phi$ such that

$$
\phi(z) \equiv \alpha f(z)+\beta g(z), \quad \alpha, \beta \geqq 0, \quad \alpha+\beta=1,
$$

where $f$ and $g$ are in $\mathcal{F}_{1}$. Given the class $\mathcal{F}_{1}$, what can we say about the class $Q$ ? Perhaps the most natural question concerns the valence of $\phi$ when $\mathcal{F}_{1}=\mathcal{V}_{1}(1)$. For some time I thought that the valence of $\phi$ must be 1 or 2 , and it was only after giving up this erroneous opinion, that I proved [24] that for a suitable choice of $f$ and $g$ in $V_{1}(1)$, the function $\phi=(f+g) / 2$ has infinite valence in $\varepsilon$. The very same selection also gives a function

$$
G(z) \equiv(f(z) g(z))^{1 / 2}
$$

that has infinite valence in $\mathcal{E}$. Thus there is an infinite sequence of constants $R(p)<1, p=1,2, \cdots$, such that if $f$ and $g \in V_{1}(1)$ and $\phi \in Q$, then $\phi$ has valence $q \leqq p$ in $|z|<R(p)$, where $R(p)$ is the largest such number with this property. $R(p)$ is called the radius of $p$-valence for the class $a$. A similar sequence of constants exists for the class $\mathcal{G}$ defined by (9.3). Except for $R(1)$, the values of $R(p)$ in these two sequences are at present unknown.

Other selections for the set $\mathfrak{F}_{1}$, or other choices for the property of $\phi$ or $G$, generate further interesting problems, most of which are open. A particular case is the problem suggested by Hayman $[31$, p. 38], namely, if $f$ and $g$ are in $\mathcal{K}_{1}(1)$ is it true that $\phi(z)$ is in $S J^{(0)}(1)$ ? In pursuing this problem MacGregor [44] found that $\phi(z)$ need not be univalent, and he determined the radius of univalence in three interesting cases:

(A) If $\digamma_{1}=V_{1}(1)$, then $R(1)=\sin (\pi / 8)=\left(2-2^{1 / 2}\right)^{1 / 2} / 2$.

(B) If $\Im_{1}=\mathfrak{K}_{1}(1)$, then $R(1)=2^{1 / 2} / 2$.

(C) If $F_{1}=S J^{(0)}(1)$, then $R(1)$ is the largest value of $r$, such that $R\left((1+z) /(1-z)^{3}\right) \geqq 0$ for $|z| \leqq r$.

Further J. S. Ratti [53] has proved that if $\mathcal{F}_{1}=\mathfrak{K}_{1}(1)$, then $R_{8}$ $=2^{1 / 2} / 2$ for the class $Q$. However, $R_{8}$ for $Q$ is unknown if $F_{1}=V_{1}(1)$ or if $\digamma_{1}=S J_{1}^{(0)}(1)$. Further $R_{c}$ is unknown $n^{4}$ for the set $Q$, for each of the three selections for $\mathfrak{F}_{1}$.

4 After this paper was completed, I learned that G. LaBelle and Q. I. Rahman have proven that if $\mathscr{F}_{1}=\mathcal{K}_{1}(1)$, then $R_{0}$ is not less than the smallest positive root of $1-3 r+2 r^{2}-2 r^{8}=0$. 
It seems to me that if $\mathcal{F}_{1}=\mathfrak{K}_{1}(1)$, then $\phi(z)$, defined by (9.2), cannot have valence 3 , so that for this family $R(2)=R(3)=\cdots=1$. More generally if $\alpha_{k}>0$, and $f_{k}(z) \in \mathcal{K}_{1}(1), k=1,2, \cdots, n$, then I conjecture that the function

$$
\phi(z) \equiv \alpha_{1} f_{1}(z)+\alpha_{2} f_{2}(z)+\cdots+\alpha_{n} f_{n}(z)
$$

has valence $p \leqq n$. If this is true, then the result is sharp, because there are $n$ functions $f_{k}$ in $\mathscr{K}_{1}(1)$ for which $\phi(z)$, given by (9.4), is $n$-valent.

Further results on the sums (9.2) and (9.4) were obtained by B. N. Rahmanov [49], [50], [52], but limitations of space forbid a detailed report.

We now consider the class $g$ defined by (9.3) with $f$ and $g$ in $\mathcal{F}_{1}$. If $F_{1}=\mho_{1}(1)$, then $\mathcal{G}$ contains functions that have valence infinity. If $F_{1}=S J_{1}^{(0)}(1)$ then, as almost everyone seems to know, $G(z) \in S J_{i}^{(0)}(1)$. For the class $\mathcal{G}$, Ratti [53] has proved:

(A) if $\mathcal{F}_{1}=V_{1}(1)$, then $R_{s}=\tanh (\pi / 4)$, (B) if $\mathcal{F}_{1}=\delta J_{1}^{(0)}(1)$, then $R_{c}=2-3^{1 / 2}$, and $(\mathrm{C})$ if $\digamma_{1}=\mathfrak{K}_{1}(1)$, then $R_{c}=\left(2(3)^{1 / 2}-3\right)^{1 / 2}$.

10. Other operations. Let $\mathcal{H C}$ be the class of functions

$$
H(z)=z+\sum_{n=2}^{\infty} a_{n} b_{n} z^{n}
$$

where $f(z)$, given by $(9.1)$, is in $\digamma_{1}$, and

$$
g(z)=z+\sum_{n=2}^{\infty} b_{n} z^{n}
$$

is also in $\mathcal{F}_{1}$. Just as in $\$ 9$, we may search for properties of the class $\mathcal{H}$.

M. S. Robertson [54] has proved that (A) if $\Im_{1}=\Im_{1}(1)$, then $H(z)$ is typically real in $|z|<2-3^{1 / 2}$ and this result is sharp, and (B) if $f$ and $g$ are univalent and convex in the direction of the imaginary axis, then $H(z)$ is also. L. Zalcman [62] has proved that there is an $f$ and $g$ in $J_{1}(1)$ such that $H(z)$ has valence infinity. This raises a host of open questions: namely, find $R(p)$, the radius of $p$-valence for the class $\mathcal{H C}_{\mathrm{C}}$ when $\digamma_{1}$ is $\mathcal{V}_{1}(1)$, or $S J_{1}^{(0)}(1)$, or $\digamma_{1}(1)$, or $\mathfrak{K}_{1}(1)$.

Similar questions can be raised about the related class $\mathcal{F}^{\star}$ of functions

$$
H^{\star}(z)=z+\sum_{n=2}^{\infty} \frac{a_{n} b_{n}}{n} z^{n}
$$


when $f$ and $g$, given by (9.1) and (10.2) are in $\Im_{1}$. Here Robertson [54] has proved that if $\Im_{1}=\Im_{1}(1)$, then $\Im^{\star}=\Im_{1}(1)$. It was suggested by several mathematicians that the set $\nu_{1}(1)$ of univalent functions might be closed under the operation (10.3), but this conjecture was killed simultaneously by B. Epstein and I. J. Schoenberg [12], C. Loewner and E. Netanyahu [41], and W. K. Hayman [29]. This naturally raises the question of determining the maximum valence for functions in the class $\mathcal{F}^{\star}$, and the radius of $p$-valence for the class $\mathfrak{K}^{\star}$. Further one can raise the same questions when $\Im_{1}=V_{1}(q)$ for $p>q \geqq 2$.

M. Biernacki [7] attempted to prove that if $f(z) \in \mathcal{V}(1)$, then

$$
g(z) \equiv \int_{0}^{z} \frac{f(t)}{t} d t
$$

is also in $\mathcal{V ( 1 ) . ~ U n f o r t u n a t e l y , ~ h i s ~ w o r k ~ c o n t a i n e d ~ a n ~ e r r o r , ~ a s ~ w a s ~}$ pointed out by J. Krzyz and Z. Lewandowski [37] who gave a counterexample. The exact nature of the Biernacki error has been explored by V. A. Pohilevič [47], [48], who does not agree with the analysis given by Krzyz and Lewandowski. But the counterexample automatically poses the problem of finding the radius of $p$-valence for the class of functions defined by (10.4), not only when $f$ is in $V(1)$, but more generally if $f$ is in $V(q)$.

R. Libera [39] proved that if $f(z) \in \delta J^{(0)}(1)$, then

$$
g(z) \equiv \frac{2}{z} \int_{0}^{z} f(t) d t
$$

is in $\delta \Im^{(0)}(1)$. Further if $f(z) \in \mathcal{K}(1)$, then $g(z)$ given by $(10.5)$ is also in $\Re(1)$. He also proved that if $f(z)$ is in $\delta J^{(0)}(1)$ then

$$
\sigma(z) \equiv \int_{0}^{z} f(t) d t
$$

is in $\$ J^{(\theta)}(2)$.

In this same direction, MacGregor [43] showed that there is an $f(z)$ in $\vartheta(1)$ such that for each $\alpha$ in $(0,2 \pi)$, the function

$$
g(z, \alpha) \equiv \frac{1}{\alpha} \int_{0}^{\alpha} f\left(z e^{i \theta}\right) d \theta
$$

is not univalent in $\varepsilon$.

11. Miscellaneous items. It is an easy matter to prove that if 


$$
\sum_{k=2}^{\infty} k\left|a_{k}\right| \leqq 1
$$

then $f(z)$, defined by (9.1) is univalent in $\mathcal{E}$. If the condition (11.1) is satisfied for $f(z)$, then it is also satisfied for each of its partial sums

$$
\sigma_{n}(z)=z+\sum_{k=2}^{n} a_{k} z^{k}, \quad n \geqq 2 .
$$

Hence, if $f(z)$ satisfies (11.1), then $\sigma_{n}(z)$ is univalent in $\varepsilon$ for each $n \geqq 2$. J. S. Ratti [53] has raised the following question: What is the class $P S_{1}$ of all functions $f(z)=z+\cdots$ such that each of the partial sums of $f(z)$ is univalent in $\varepsilon$ ? Obviously $P S_{1}$ contains all functions that satisfy the condition (11.1), but it is certainly a larger class, since $f_{0}(z) \equiv z+z^{2} / 2+z^{3} / 12$ is in $P S_{1}$ and does not satisfy (11.1).

Although a polynomial is more elementary than an infinite power series, sharp results for univalent polynomials are harder to obtain than the corresponding results for univalent functions. The reader should observe that if $|b|<1$, then the transformation

$$
g(z) \equiv[f((z+b) /(1+b z))-f(b)] / f^{\prime}(b)\left(1-|b|^{2}\right)
$$

takes a function $f(z)$ in $V_{1}(1)$ into a function $g(z)$ that is also in $V_{1}(1)$. But if $f(z)$ is a univalent polynomial, $g(z)$ will not be another polynomial.

A function $f(z)$ in $v_{1}(1)$ is said to be bi-univalent if the inverse function is also univalent in $\mathcal{E}$. Almost nothing is known about biunivalent functions aside from the inequality $\left|a_{2}\right|<1.51$ obtained by M. Lewin [38].

\section{REFERENCES}

1. H. Actilov, On certain questions of the theory of univalent conformal mapping, Izv. Akad. Nauk UzSSR Ser. Fiz.-Mat. Nauk 10 (1966), no. 5, 3-9. (Russian)

2. L. A. Aksent'ev, On integral representations of univalent functions, Izv. Vysక. Učebn. Zaved. Matematika 1959, no. 4 (11), 3-8. (Russian)

3. J. Bender, Some extremal theorems for multivalently star-like functions, Duke Math. J. 29 (1962) 101-106.

4. S. D. Bernardi, A survey of the development of the theory of schlicht functions, Duke Math. J. 19 (1952), 263-287.

5. - A bibliography of schlicht functions, New York University, Courant Institute of Mathematical Sciences, 1966.

6. M. Biernacki, Les fonctions multivalentes, Hermann, Paris, 1938.

7. - Sur l'intégrale des fonctions univalentes, Bull. Acad. Polon. Sci. Sér. Sci. Math. Astronom. Phys. 8 (1960), 29-34.

8. L. Cakalov, Sur une class de fonctions analytiques univalentes, C. R. Acad. Sci. Paris 242 (1956), 437-439. 
9. - Sur les domaines d'univalence de certaines classes de fonctions analytiques, Bŭlgar. Akad. Nauk. Izv. Mat. Inst. 4 (1960), 43-55. (Bulgarian)

10. R. J. Distler, The domain of univalence of certain classes of meromorphic functions, Proc. Amer. Math. Soc. 15 (1964), 923-928.

11. L. E. Dundučenko, A generalization of classes of analytic functions considered by L. Cakalov, Bulgar. Akad. Nauk. Izv. Mat. Inst. 5 (1961), 35-41. (Russian)

12. B. Epstein and I. J. Schoenberg, On a conjecture concerning schlicht functions, Bull. Amer. Math. Soc. 65 (1959), 273-275.

13. I. M. Gal'perin, The theory of univalent functions with bounded rotation, Izv. Vyss. Ucebn. Zaved. Matematika 1958, no. 3(4), 50-61. (Russian)

14. S. A. Gel'fer, On typically-real functions of order p, Mat. Sb. 35(77) (1954), 193-214; English transl., Amer. Math. Soc. Transl. (2) 18 (1961), 15-36.

15. - The variation of multivalent functions, Dokl. Akad. Nauk SSSR 98 (1954), 885-888; English Transl., Amer. Math. Soc. Transl. (2) 26 (1963), 1-4.

16. - On the coefficient problem for p-valent functions, Dokl. Akad. Nauk SSSR 106 (1956), 955-958; English transl., Amer. Math. Soc. Transl. (2) 26 (1963), 5-10.

17. - The method of variations in the theory of p-valent functions, Uspehi Mat. Nauk 11 (1956), no. 5 (71), 60-66; English transl., Amer. Math. Soc. Transl. (2) 18 (1961), 37-43.

18. G. M. Goluzin, Geometric theory of functions of a complex variable, GITTL, Moscow, 1952; 2nd ed., Nauka; Moscow, 1966; German transl., VEB Deutscher Verlag, Berlin, 1952.

19. A. W. Goodman, On some determinants related to p-valent functions, Trans. Amer. Math. Soc. 63 (1948), 175-192.

20. - On the Schwarz-Christoeffel transformation and p-valent functions, Trans. Amer. Math. Soc. 68 (1950), 204-223.

21. - Typically-real functions with assigned zeros, Proc. Amer. Math. Soc. 2 (1951), 349-357.

22. - On the critical points of a multivalent function, Trans. Amer. Math. Soc. 89 (1958), 295-309.

23. - A note on bivalent functions, J. London Math. Soc. 39 (1964), 215-219.

24. - The valence of sums and products, Canad. J. Math. (to appear).

25. A. W. Goodman and M. S. Robertson, $A$ class of multivalent functions, Trans. Amer. Math. Soc. 70 (1951), 127-136.

26. W. K. Hayman, Some applications of the transfinite diameter to the theory of functions, J. Analyse Math. 1 (1951), 155-179.

27. - The asymptotic behaviour of p-valent functions, Proc. London Math. Soc. (3) 5 (1955), 257-284.

28. —- Multivalent functions, Cambridge Univ. Press, Cambridge, 1958.

29. - On the coefficients of univalent functions, Proc. Cambridge Philos. Soc. 55 (1959), 373-374.

30. - Coefficient problems for univalent functions and related function classes, J. London Math. Soc. 40 (1965), 385-406.

31. - Research problems in function theory, Athlone Press, London, 1967.

32. J. A. Hummel, Multivalent starlike functions, J. Analyse Math. 18 (1967), 133160.

33. J. A. Jenkins, Univalent functions and conformal mapping, Springer-Verlag, Berlin, 1958; Russian transl., IL, Moscow, 1962. 
34. P. Koebe, Über die Uniformisierung beliebiger analytischer Kurven, Nachr Ges. Wiss. Göttingen (1907), 191-210.

35. J. Krzyz, A counterexample concerning univalent functions, Folia Societatis Scientiarum Lublinensis 2 (1962), 57-58.

36. - On some recent results in the theory of analytic functions, Colloq. Math. 11 (1964), 147-150.

37. J. Krzyz and Z. Lewandowski, On the integral of univalent functions, Bull. Acad. Polon. Sci. Sér. Sci. Math. Astronom. Phys. 11 (1963), 447-448.

38. M. Lewin, On a coefficient problem for biunivalent functions, Proc. Amer. Math. Soc. 18 (1967) 63-68.

39. R. Libera, Some classes of regular univalent functions, Proc. Amer. Math. Soc. 16 (1965), 755-758.

40. C. Loewner, Untersuchungen iiber die Verzerrung bei konformen Abbildungen des Einheitskreises, Leipzig Berichte 69 (1917), 89-106.

41. C. Loewner and E. Netanyahu, On some compositions of Hadamard type in classes of analytic functions, Bull. Amer. Math. Soc. 65 (1959), 284-286.

42. T. H. MacGregor, Functions whose derivative has a positive real part, Trans. Amer. Math. Soc. 104 (1962), 532-537.

43. - Certain integrals of univalent and convex functions, Math. Z. 103 (1968), 48-54.

44. - The univalence of a linear combination of convex mappings, J. London Math. Soc. (to appear).

45. P. Montel, Lecons sur les fonctions univalentes oi multivalentes, Gauthier-Villars, Paris, 1933.

46. R. Nevanlinna, Über die konforme Abbildung von Sterngebieten, Ofversigt av Finska Vetenskaps-Societetens Förhandinger 53 (A) (1921), $\mathrm{Nr} 6$.

47. V. A. Pohilevix, On a theorem of M. Biernacki, Dopovidi Akad. Nauk Ukrain. RSR 1965, 423-425. (Ukrainian)

48. - On a theorem of $M$. Biernacki in the theory of univalent functions, Ukrain. Mat. Ž. 17 (1965), no. 4, 63-71. (Russian)

49. B. N. Rahmanov, On the theory of univalent functions, Dokl. Akad. Nauk SSSR 88 (1953), 413-414. (Russian)

50. - On the theory of univalent functions, Dokl. Akad. Nauk SSSR 91 (1953), 729-732. (Russian)

51. - On the theory of univalent functions, Dokl. Akad. Nauk SSSR 97 (1954), 973-976; English transl., Amer. Math. Soc. Transl. (2) 32 (1963), 159-162.

52. - On the theory of univalent functions, Dokl. Akad. Nauk SSSR 103 (1955), 369-371. (Russian)

53. J. S. Ratti, Paper in preparation.

54. M. S. Robertson, Applications of a lemma of Fejêr to typically-real functions, Proc. Amer. Math. Soc. 1 (1950), 555-561.

55. - A coefficient problem for functions regular in an annulus, Canad.J. Math. 4 (1952), 407-423.

56. W. Rogosinski, Über positive harmonische Entwicklungen und typisch-reelle Potenzreihen, Math. Z. 35 (1932), 93-121.

57. K. Sakaguchi and S. Watanabe, Meromorphic functions multivalently starlike in a wide sense, J. Nara Gakugei Univ. Natur. Sci. 11 (1963), 3-7.

58. A. C. Schaeffer and D. C. Spencer, Coefficient regions for schlicht functions, Amer. Math. Soc. Colloq. Publ., vol. 35, Amer. Math. Soc., Providence, R. I., 1950. 
59. M. Schiffer, Variation of the Green function and theory of the p-valued functions, Amer. J. Math. 65 (1943), 341-360.

60. P. Todorov, On the radius of univalence of a class of meromorphic functions, Časopis Pěst Mat. 91 (1966), 77-79. (Russian)

61. M. Watson, On functions that are bivalent in the unit circle, J. Analyse Math. 17 (1966), 383-409.

62. L. Zalcman, Hadamard products of Schlicht functions, Proc. Amer. Math. Soc. 19 (1968), 544-548.

63. V. A. Zmorovic, On open problems in the theory of univalent functions, Nauk Zaniski, Kiev. Lerjeabvir Univ. 11 (1952), 83-94. (Russian)

64. - On certain special classes of analytic functions univalent in a circle, Uspehi Mat. Nauk. 9 (1954), no. 4 (62), 175-182. (Russian)

UNIVERSITY OF SOUTH FLORIDA 